\title{
Atraso na procura pelo primeiro atendimento para o diagnóstico da tuberculose*
}

\author{
DELAY IN SEEKING INITIAL CARE FOR TUBERCULOSIS DIAGNOSIS
}

\author{
DEMORA EN LA BÚSQUEDA DE LA PRIMERA CONSULTA PARA EL DIAGNÓSTICO DE \\ LA TUBERCULOSIS
}

\section{Anneliese Domingues Wysocki ${ }^{1}$, Maria Amélia Zanon Ponce ${ }^{2}$, Beatriz Estuque Scatolin ${ }^{3}$, Rubia Laine de Paula Andrade ${ }^{4}$, Silvia Helena Figueiredo Vendramini ${ }^{5}$, Antonio Ruffino Netto ${ }^{6}$, Tereza Cristina Scatena Villa ${ }^{7}$}

\section{RESUMO}

Estudo descritivo que objetivou analisar o tempo decorrido entre a percepção dos sintomas da tuberculose (TB) e a primeira busca por serviço de saúde segundo características dos doentes com TB em São José do Rio Preto - SP. Entrevistaram-se 97 doentes com TB utilizando instrumento estruturado. Identificou-se atraso do doente pela mediana do tempo entre a percepção dos sintomas e busca por atendimento (> 15 dias). Calculou-se a razão de prevalência para identificar variáveis relacionadas ao atraso. Houve atraso entre: doentes do sexo masculino, com 18 a 29 e 50 a 59 anos, baixa escolaridade, maior renda familiar, casos pulmonares, não coinfectados com HIV, sintomas fracos, consumidores de bebidas alcoólicas e tabaco, que não realizavam controle preventivo de saúde e procuravam o serviço de saúde mais próximo do domicílio. O reconhecimento do perfil dos usuários na busca por atendimento é primordial para definir estratégias que favoreçam a utilização dos serviços em momento oportuno.

\section{DESCRITORES}

Tuberculose

Diagnóstico tardio

Acesso aos Serviços de Saúde

Aceitação pelo paciente de cuidados de saúde

\begin{abstract}
Descriptive study, which aimed to analyze the time between the perception of TB symptoms onset and the first search for health services, according to TB patient characteristics in São José do Rio Preto - SP. 97 TB patients in treatment were interviewed with a structured instrument. The delay was identified by the median time between the perception of TB symptom's onset and the patients demand for care (> 15 days). To identify the variables related to delay the prevalence ratio were calculated. There was a delay seeking care among: male patients, aged 18 to 29 and 50 to 59 years, lower education and higher family income, pulmonary cases without HIV co-infection, presenting milder symptoms, users of alcoholic beverages and tobacco, those who did not perform preventive health control, and who sought health services closer to home. The recognition of users' profiles in the search for care is paramount for defining strategies that favor the use of services in a timely manner.
\end{abstract}

\author{
DESCRIPTORS \\ Tuberculosis \\ Delayed diagnosis \\ Health Services Accessibility \\ Patient acceptance of health care
}

\begin{abstract}
RESUMEN
Estudio descriptivo objetivando analizar el tiempo transcurrido entre la percepción de los primeros síntomas de tuberculosis (TB) y la búsqueda de una primera consulta en servicios de salud, según características de los enfermos de TB en São José de Rio Preto-SP. Fueron entrevistados 97 enfermos de TB, utilizándose instrumento estructurado. Se identificó atraso del enfermo por la mediana de tiempo entre percepción de síntomas y búsqueda de atención (>15 días). Se calculó la razón prevalente para identificar variables relacionadas al atraso. Existió atraso entre: enfermos masculinos, con 18 a 29 y 50 a 59 años, baja escolarización, mayor renta familiar, casos pulmonares, no coinfectados con HIV, síntomas débiles, consumidores de alcohol y tabaco, sin control sanitario preventivo, buscando servicio de salud próximo al domicilio. El reconocimiento del perfil de pacientes buscando atención es primordial para definir estrategias que favorezcan la utilización de los servicios en el momento oportuno.
\end{abstract}

\author{
DESCRIPTORES \\ Tuberculosis \\ Diagnóstico tardío \\ Accesibilidad a los Servicios de Salud \\ Aceptación de la Atención de Salud
}

\footnotetext{
* Extraído da dissertação "Tuberculose: tempo decorrido entre o início dos sintomas e a procura pelo Serviço de Saúde em São José do Rio Preto", Programa de Enfermagem Materno Infantil e Saúde Pública da Escola de Enfermagem de Ribeirão Preto da Universidade de São Paulo, $2011 .{ }^{1}$ Enfermeira. Mestre em Ciências da Saúde. Doutoranda pelo Programa de Pós-Graduação Interunidades em Enfermagem da Escola de Enfermagem de Ribeirão Preto da Universidade de São Paulo. Ribeirão Preto, SP, Brasil. lilise@usp.br ${ }^{2}$ Enfermeira. Doutoranda pelo Programa de Pós-Graduação em Enfermagem Materno Infantil e Saúde Pública da Escola de Enfermagem de Ribeirão Preto da Universidade de São Paulo. Ribeirão Preto, SP, Brasil. amelinha famerp@yahoo.com.br ${ }^{3}$ Enfermeira. Mestre em Ciências da Saúde. Doutoranda pelo Programa de Pós-Graduação Interunidades em Enfermagem da Escola de Enfermagem de Ribeirão Preto da Universidade de São Paulo. Ribeirão Preto, SP, Brasil. scatolin@eerp.usp.br ${ }^{4}$ Enfermeira. Doutoranda pelo Programa de Pós-Graduação Interunidades em Enfermagem. Especialista em Laboratório da Escola de Enfermagem de Ribeirão Preto da Universidade de São Paulo. Ribeirão Preto, SP, Brasil. rubia@eerp.usp.br ${ }^{5}$ Enfermeira. Doutora. Professora da Faculdade de Medicina de São José do Rio Preto. São José do Rio Preto, SP, Brasil. silviahve@gmail.com ${ }^{6}$ Professor Titular da Faculdade de Medicina de Ribeirão Preto da Universidade de São Paulo. Ribeirão Preto, SP, Brasil. aruffino@fmrp.usp.br ${ }^{7}$ Professora Titular da Escola de Enfermagem de Ribeirão Preto da Universidade de São Paulo. Ribeirão Preto, SP, Brasil. tite@eerp.usp.br
} 


\section{INTRODUÇÃO}

O controle da tuberculose (TB) prevê, entre suas principais atividades, a eliminação da fonte de infecção na comunidade como aspecto fundamental para diminuir a morbidade e mortalidade e interromper a cadeia de transmissão do agravo ${ }^{(1)}$. No cenário mundial, no ano de 2009, a incidência notificada da TB foi de 140 casos/100.000 habitantes, sendo que no Brasil foram notificados 71,7 mil casos novos, com uma incidência estimada de 45 casos/100.000 habitantes, fazendo com que o País ocupasse o 190 lugar no ranking dos 22 países de maior incidência da TB e o 13ㅇ lugar em número de casos bacilíferos ${ }^{(1)}$.

Estudos têm mostrado que as elevadas taxas de incidência e prevalência da TB são resultantes não só das condições que possibilitam vulnerabilidade ao adoecimento, mas também da demora em realizar o diagnóstico da doença ${ }^{(2-5)}$. Nesse sentido, o diagnóstico precoce configura-se como um desafio, considerando as dificuldades relacionadas tanto à organização dos serviços de saúde, dada a precariedade de integração das ações prioritárias e a utilização de práticas fragmentadas que limitam o acesso à oferta de serviços em saúde ${ }^{(6)}$, quanto aos aspectos socioculturais, econômicos e geográficos intrínsecos aos doentes com TB que determinam a busca, utilização e aceitação da atenção proporcionada. Tais questões são vistas como possíveis obstáculos à efetividade dos programas e serviços de saúde uma vez que os resultados dos indicadores em saúde são obtidos a partir do momento em que os serviços estão acessíveis e os usuários aceitam, utilizam e dão continuidade à sua assistência ${ }^{(3)}$.

Dada a importância da contribuição dos doentes para o atraso no diagnóstico da TB, estudos internacionais ${ }^{(3,5)}$ vêm tentando desvelar os aspectos que contribuem para a utilização tardia dos serviços de saúde e que, portanto, configuram-se como barreira para a obtenção do diagnóstico em tempo oportuno. Aspectos relacionados ao conhecimento que o doente tem sobre a doença, distância entre sua moradia e a unidade de saúde, custos relacionados ao transporte, impossibilidade de faltar ao trabalho e compromissos, bem como a satisfação com o atendimento prestado nos serviços de saúde e a procura por cuidados alternativos são apontados como determinantes para o acesso ao diagnóstico da doença ${ }^{(3-5)}$. No Brasil, no entanto, pouco se sabe acerca dos aspectos comportamentais intrínsecos ao doente com TB e os aspectos que o influenciam na busca por atendimento e obtenção diagnóstica, bem como o tempo dispendido nesse processo. Utilizando os descritores Diagnóstico tardio e Tuberculose, identificaram-se 79 estudos em uma busca sistematizada na Biblioteca Virtual em Saúde (BVS) sem especificação do ano de publicação. Desses, no entanto, apenas três foram desenvolvidos no Brasil e somente um deles analisou os aspectos relacionados ao atraso dos doentes com TB para buscar por atendimento de saúde após a percepção dos sintomas da doença $a^{(2,7-8)}$.

Diante da magnitude da TB como problema de saúde pública e da importância do diagnóstico precoce da doença como uma das condições para o seu controle, este estudo teve como objetivo analisar o tempo decorrido entre a percepção do início dos sintomase a primeira procura pelo serviço de saúde segundo características sociodemográficas, clínicas, econômicas e comportamentais dos doentes com TB.

\section{MÉTODO}

Trata-se de um estudo epidemiológico descritivo tipo transversal, realizado no ano de 2009 no município de São José do Rio Preto. No referido ano, a rede municipal de atenção à $T B$ estava organizada de forma regionalizada em cinco distritos sanitários. No nível primário de atenção, o município possuía 13 Unidades Básicas de Saúde (UBS), 11 Unidades de Saúde da Família (USF) e cinco Unidades de Pronto Atendimento (UPA). No nível secundário, um Ambulatório Regional de Especialidades (NGA-60) com Programa de Controle da Tuberculose (PCT) e um Ambulatório de aids. Já no nível terciário existiam seis hospitais.

A porta de entrada dos suspeitos de TB para o diagnóstico da doença no município de estudo pode ser qualquer ponto de atenção à saúde e, uma vez diagnosticados, os doentes são encaminhados para os serviços de Atenção Básica para o seu manejo clínico e de seus comunicantes. Nesse nível de atenção, o doente com TB é acompanhado por uma equipe mínima generalista de profissionais composta por Médico, Enfermeiro e Auxiliar/técnico de enfermagem, que atuam com suporte técnico oferecido pela equipe especializada do PCT. O acompanhamento dos casos de TB que exigem maior atenção, como doentes com intoxicações medicamentosas decorrentes do tratamento, micobactérias atípicas e multidrogarresistentes (MDR), é realizado diretamente pelas equipes especialistas do PCT e ambulatório de aids (coinfectados).

Cabe destacar a importância do papel do enfermeiro na gestão dos casos de TB no município, sendo este o profissional que assume a coordenação do cuidado, realiza a articulação com os demais níveis de atenção e mantém o vínculo necessário com os usuários, favorecendo a participação dos mesmos no decorrer do tratamento.

A população de estudo foi composta por doentes com TB que realizaram tratamento no município no período 
de novembro de 2008 a novembro de 2009. Nesse período, após o levantamento do número de doentes nas bases de dados do sistema estadual de informações em TB (TB-WEB), encontraram-se 135 doentes em tratamento; entretanto, desses, apenas 110 atendiam aos critérios de inclusão do estudo, sendo excluídos: 12 doentes menores de 18 anos, três detentos, dois doentes psiquiátricos e oito mudanças de diagnóstico. Uma vez que nove doentes se recusaram a participar da pesquisa e um não tinha condições de responder por estar internado na Unidade de Terapia Intensiva, um total de 100 doentes foram entrevistados, porém, 97 constituíram a população final do estudo, uma vez que dois foram excluídos por não saberem informar o tempo dispendido entre a percepção do início dos sintomas da TB até a procura pelo primeiro atendimento e um não soube informar o primeiro serviço de saúde procurado.

Os dados foram coletados no período de julho a dezembro de 2009 por meio de fontes primárias (entrevistas) e de fontes secundárias (consultas a prontuários e sistema de informação TB-Web). Para as entrevistas utilizou-se um instrumento de coleta de dados estruturado que buscou recuperar o trajeto do doente desde o momento em que percebeu o início dos sintomas da TB até a procura pelo primeiro atendimento nos serviços de saúde. A aplicação do questionário ocorreu no domicílio dos doentes ou nos serviços de saúde que os acompanhavam.

Para a análise dos dados, inicialmente foi utilizada a informação fornecida pelos doentes com TB em relação ao tempo (dias) que eles levaram entre perceber-se com os sintomas e procurar pelo primeiro atendimento em um serviço de saúde, denominando essa variável de tempo do doente. Destaca-se que essa informação refere-se à necessidade percebida pelo doente em buscar por atendimento a partir da autoavaliação do seu estado de saúde, o que não significa que os sintomas tenham iniciado exatamente quando o doente teve a iniciativa de procurar por um serviço de saúde.

Em seguida, pelo fato de não haver uma definição consensual do tempo esperado para o doente com TB buscar por atendimento após perceber-se com os sintomas da doença ${ }^{(3)}$, neste estudo, definiu-se atraso do doente na busca por atendimento a partir de um ponto de corte para a variável tempo do doente. Para isso, calculou-se a mediana para essa variável e, a partir do cálculo, considerou-se atraso aqueles cujo tempo para procurar pelo primeiro atendimento foi superior a 15 dias, identificando assim dois grupos: com e sem atraso (variáveis dependentes). Optou-se pela utilização da mediana uma vez que a distribuição dos valores referentes às respostas dos doentes para a variável tempo é assimétrica, possibilitando a amenização da interferência de valores aberrantes.

A razão de prevalência (RP) (Figura 1) foi calculada para identificar quais as categorias das variáveis independentes selecionadas estariam associadas ao atraso do doente na busca por atendimento. Padronizou-se como numerador a primeira categoria de cada variável. Foram construídos intervalos de confiança (IC 95\%) adotando nível de significância de $5 \%$.

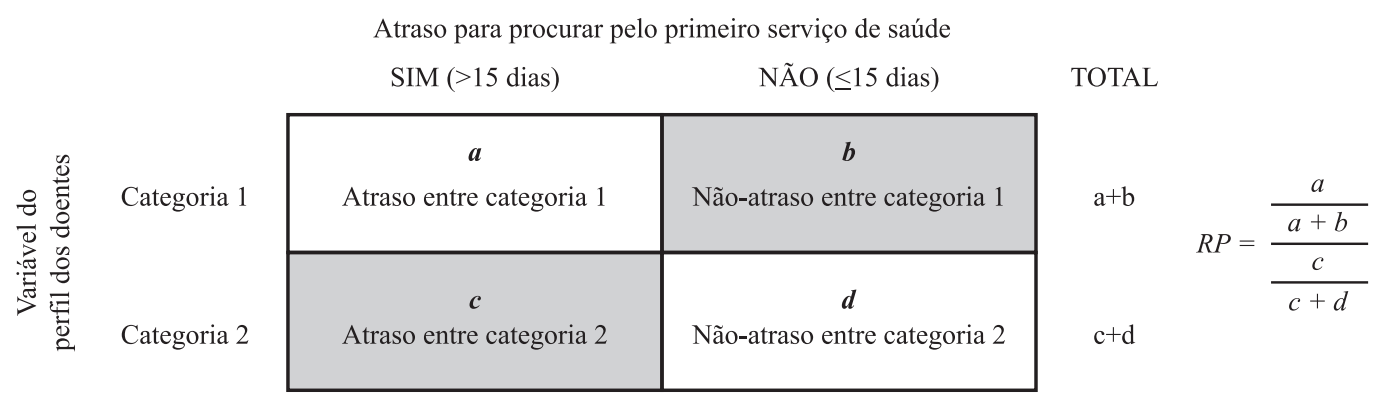

Figura 1 - Esquema proposto para o cálculo da Razão de Prevalência, segundo o atraso para buscar pelo primeiro serviço de saúde e variáveis do perfil dos doentes - São José do Rio Preto, 2009

As variáveis escolhidas para analisar o atraso do doente foram selecionadas segundo características sociodemográficas e econômicas (sexo, faixa etária, estado civil, escolaridade, renda familiar, situação empregatícia), clínicas (forma clínica da doença, tipo de caso, coinfecção TB/ $H I V$, percepção sobre a intensidade dos sintomas da TB) e comportamentais (consumo de tabaco e bebidas alcoólicas, realização de controle preventivo de saúde (antes da $T B$ ), procura do serviço de saúde mais próximo do domicílio (antes da TB), primeiro serviço de saúde procurado ao início dos sintomas da TB, conhecimento sobre a TB antes do diagnóstico) dos indivíduos adoecidos por TB.
Ressalta-se que informações acerca da intensidade dos sintomas da TB e do conhecimento sobre a TB antes do diagnóstico foram obtidas a partir da autopercepção dos doentes sobre questões com categorias de respostas preestabelecidas (sintomas fracos ou fortes e conhecimento satisfatório ou precário), não sendo utilizadas outras medidas de aferição.

Atendendo à resolução no 196/96, o projeto foi submetido e aprovado pelo Comitê de Ética da Faculdade de Medicina de São José do Rio Preto, conforme protocolo no $7081 / 2008$.
Atraso na procura pelo primeiro atendimento para o diagnóstico da tuberculose Wysocki AD, Ponce MAZ, Scatolin BE, Andrade RLP, Vendramini SHF, Ruffino-Netto A, Villa TCS 


\section{RESULTADOS}

A mediana do tempo decorrido entre o início dos sintomas da TB e a procura pelo primeiro atendimento em um serviço de saúde foi de 15 dias, com intervalo inter- quartil de 4-45 dias (Figura 2). Observa-se que em alguns casos os doentes excederam 90 dias.

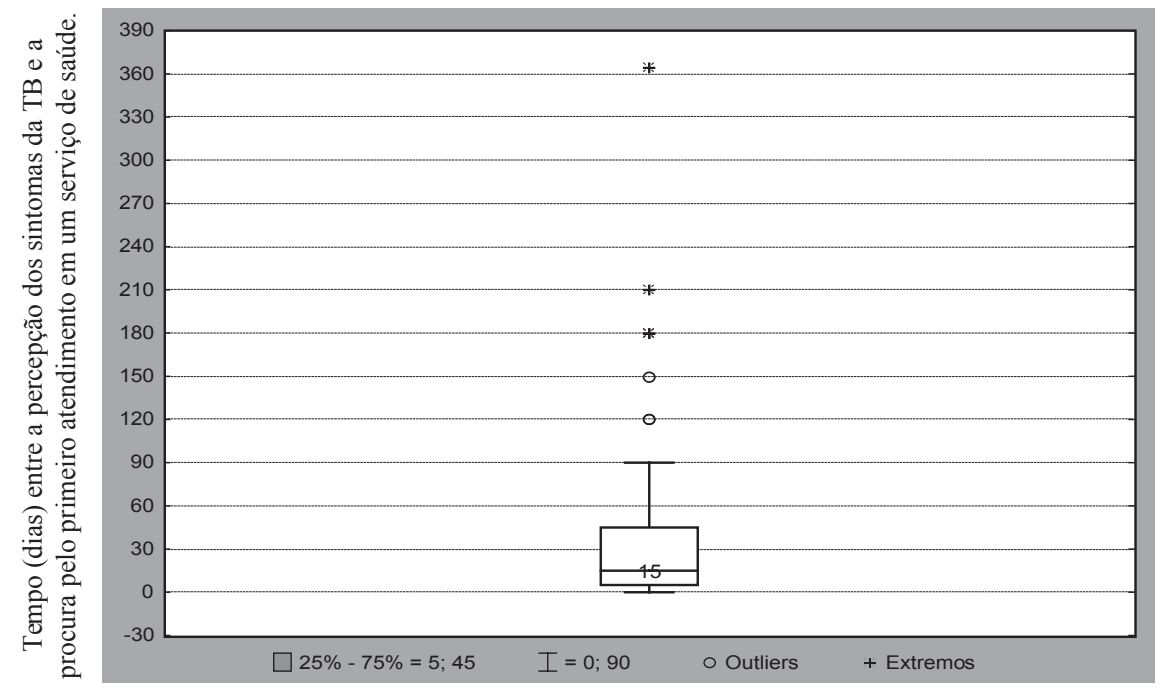

Figura 2 - Tempo (dias) decorrido entre a percepção do início dos sintomas da TB e a procura pelo primeiro atendimento nos serviços de saúde - São José do Rio Preto, 2009

Houve predominância do sexo masculino, que apresentou maior atraso na procura pelo primeiro atendimento. Doentes de 18 a 29 anos tiveram maior atraso em relação a todas as faixas etárias, com exceção dos doentes cuja faixa etária estava entre 50 e 59 anos. Atrasaram para procurar pelo primeiro atendimento doentes solteiros, em relação aos casados e viúvos, com baixa escolaridade e com maior renda familiar. Encontrou-se diferença estatisticamente significante entre a renda familiar e o atraso na busca por atendimento, e os doentes com TB que possuíam renda familiar maior que cinco Salários Mínimos (SM) tiveram maior atraso (Tabela 1).

Tabela 1 - Razão de prevalência do atraso relacionado ao doente, segundo variáveis sociodemográficas e econômicas dos doentes com TB - São José do Rio Preto, 2009

\begin{tabular}{|c|c|c|c|c|c|c|}
\hline \multicolumn{2}{|c|}{$\begin{array}{c}\text { Variáveis sociodemográficas e econômicas dos } \\
\text { doentes com TB }\end{array}$} & \multirow{2}{*}{$\begin{array}{c}\text { Atraso } \\
15\end{array}$} & \multirow{2}{*}{$\begin{array}{c}\text { Não Atraso } \\
21\end{array}$} & \multirow{3}{*}{$\begin{array}{c}\begin{array}{c}\text { TOTAL } \\
\mathbf{( N = 9 7 )}\end{array} \\
36 \\
61\end{array}$} & \multirow{3}{*}{$\frac{\mathbf{R P} *}{0,91}$} & \multirow{3}{*}{$\frac{\text { IC }}{[0,57 ; 1,46]}$} \\
\hline \multirow{2}{*}{ Sexo } & Feminino & & & & & \\
\hline & Masculino & 28 & 33 & & & \\
\hline \multirow{5}{*}{$\begin{array}{l}\text { Faixa etária } \\
\text { (em anos) }\end{array}$} & 18 a $29^{(1)}$ & 9 & 8 & 17 & \multirow{5}{*}{$\begin{array}{l}\mathrm{RP}_{1 / 2}-1,47 \\
\mathrm{RP}_{1 / 3}-1,47 \\
\mathrm{RP}_{1 / 4}-0,88 \\
\mathrm{RP}_{1 / 5}-1,13\end{array}$} & \multirow{5}{*}{$\begin{array}{l}{[0,74 ; 2,93]} \\
{[0,74 ; 2,93]} \\
{[0,48 ; 1,62]} \\
{[0,56 ; 2,29]}\end{array}$} \\
\hline & 30 a $39^{(2)}$ & 9 & 16 & 25 & & \\
\hline & 40 a $49^{(3)}$ & 9 & 16 & 25 & & \\
\hline & 50 a $59^{(4)}$ & 9 & 6 & 15 & & \\
\hline & Acima de $60^{(5)}$ & 7 & 8 & 15 & & \\
\hline \multirow{4}{*}{ Estado civil } & Solteiro ${ }^{(1)}$ & 17 & 19 & 36 & \multirow{4}{*}{$\begin{array}{l}\mathrm{RP}_{1 / 2}-1,10 \\
\mathrm{RP}_{1 / 3}-0,94 \\
\mathrm{RP}_{1 / 4}-1,42\end{array}$} & \multirow{4}{*}{$\begin{array}{l}{[0,67 ; 1,80]} \\
{[0,46 ; 1,92]} \\
{[0,53 ; 3,80]}\end{array}$} \\
\hline & Casado $^{(2)}$ & 18 & 24 & 42 & & \\
\hline & Divorciado $^{(3)}$ & 5 & 5 & 10 & & \\
\hline & Viúvo ${ }^{(4)}$ & 3 & 6 & 9 & & \\
\hline \multirow{2}{*}{ Escolaridade } & $\begin{array}{l}\text { Sem escolaridade ou EF } \\
\text { incompleto }\end{array}$ & 32 & 37 & 69 & \multirow{2}{*}{1,18} & \multirow{2}{*}[0,70;2,00]{} \\
\hline & EF completo ou mais & 11 & 17 & 28 & & \\
\hline \multirow{2}{*}{$\begin{array}{l}\text { Situação } \\
\text { empregatícia }\end{array}$} & Com remuneração & 30 & 37 & 67 & \multirow{2}{*}{1,03} & \multirow{2}{*}[0,63;1,68]{} \\
\hline & Sem remuneração & 13 & 17 & 30 & & \\
\hline \multirow{3}{*}{$\begin{array}{l}\text { Renda Familiar } \\
\text { (em Salários } \\
\text { Mínimos) }\end{array}$} & Sem rendimento até $2^{(1)}$ & 20 & 34 & 54 & \multirow{3}{*}{$\begin{array}{l}\mathrm{RP}_{1 / 2}-0,65 \\
\mathrm{RP}_{1 / 3}-0,20\end{array}$} & \multirow{3}{*}{$\begin{array}{r}{[0,41 ; 1,02]} \\
{[\mathbf{0 , 1 4 ; 0 , 2 8}]}\end{array}$} \\
\hline & De 2 a $5^{(2)}$ & 20 & 15 & 35 & & \\
\hline & Maior que $5^{(3)}$ & 3 & 5 & 8 & & \\
\hline
\end{tabular}

${ }^{*} \mathrm{RP} 1 / \mathrm{n}$ equivale a razão de prevalência entre a categoria 1 em relação a categoria $n$, sendo $n$ variando de 2 a 5 . 
Doentes com TB pulmonar, classificados como recidiva, não coinfectados com HIV e com sintomas fracos tiveram maior atraso na busca pelos serviços de saúde (Tabela 2).

Tabela 2 - Razão de prevalência do atraso relacionado ao doente, segundo variáveis clínicas dos doentes com TB - São José do Rio Preto, 2009

\begin{tabular}{|c|c|c|c|c|c|c|}
\hline \multicolumn{2}{|c|}{ Variáveis clínicas dos doentes com TB } & \multirow{2}{*}{$\frac{\text { Atraso }}{36}$} & \multirow{2}{*}{$\begin{array}{c}\begin{array}{c}\text { Não } \\
\text { Atraso }\end{array} \\
43\end{array}$} & \multirow{2}{*}{$\begin{array}{c}\text { TOTAL } \\
(\mathbf{N}=97)\end{array}$} & \multirow{3}{*}{$\begin{array}{l}\mathbf{R P} * \\
1,17\end{array}$} & \multirow{3}{*}{$\frac{\text { IC }}{[0,63 ; 2,19]}$} \\
\hline \multirow{2}{*}{ Forma clínica da TB } & Pulmonar & & & & & \\
\hline & Extrapulmonar & 7 & 11 & 18 & & \\
\hline \multirow{2}{*}{ Tipo de caso } & Casos novos & 39 & 51 & 90 & \multirow{2}{*}{0,76} & \multirow{2}{*}[0,38;1,50]{} \\
\hline & Recidiva/retratamento & 4 & 3 & 7 & & \\
\hline \multirow{2}{*}{ Coinfecção TB/HIV } & Sim & 6 & 11 & 17 & \multirow{2}{*}{0,76} & \multirow{2}{*}[0,38;1,51]{} \\
\hline & Não & 37 & 43 & 80 & & \\
\hline \multirow{2}{*}{$\begin{array}{l}\text { Percepção sobre a intensidade } \\
\text { dos sintomas da TB }\end{array}$} & Forte & 30 & 41 & 71 & \multirow{2}{*}{0,85} & \multirow{2}{*}{0,$53 ; 1,35$} \\
\hline & Fraco & 13 & 13 & 26 & & \\
\hline
\end{tabular}

*RP1/n equivale a razão de prevalência entre a categoria 1 em relação a categoria $n$, sendo $n$ variando de 2 a 5 .

Aqueles doentes com TB que se declararam consumidores de bebidas alcoólicas e tabaco, que não realizavam controle preventivo de saúde, que procuravam o serviço de saúde mais próximo do domicílio antes da TB e que declararam ter conhecimento satisfatório sobre a TB atrasaram mais para buscar por atendimento nos serviços de saúde, especialmente os que optaram por buscar os serviços de Atenção (Tabela 3).

Tabela 3 - Razão de prevalência do atraso relacionado ao doente, segundo variáveis comportamentais dos doentes com TB - São José do Rio Preto, 2009

\begin{tabular}{|c|c|c|c|c|c|c|}
\hline Variáveis comportamenta & dos doentes com TB & Atraso & $\begin{array}{c}\text { Não } \\
\text { Atraso }\end{array}$ & $\begin{array}{l}\text { TOTAL } \\
(\mathbf{N}=97)\end{array}$ & $\mathbf{R P *}$ & IC \\
\hline \multirow{2}{*}{ Consumo de bebidas alcoólicas } & Sim & 33 & 38 & 71 & \multirow{2}{*}{1,21} & \multirow{2}{*}[0,70;2,09]{} \\
\hline & Não & 10 & 16 & 26 & & \\
\hline \multirow{2}{*}{ Consumo de tabaco } & Sim & 35 & 24 & 59 & \multirow{2}{*}{1,19} & \multirow{2}{*}[0,81;1,74]{} \\
\hline & Não & 19 & 19 & 38 & & \\
\hline \multirow{2}{*}{$\begin{array}{l}\text { Realização de controle } \\
\text { preventivo de saúde antes da TB }\end{array}$} & Sim & 30 & 41 & 71 & \multirow{2}{*}{0,85} & \multirow{2}{*}[0,53;1,35]{} \\
\hline & Não & 13 & 13 & 26 & & \\
\hline \multirow{2}{*}{$\begin{array}{l}\text { Procurou pelo serviço de saúde } \\
\text { mais próximo do domicílio }\end{array}$} & Sim & 35 & 40 & 75 & \multirow{2}{*}{1,28} & \multirow{2}{*}[0,70;2,35]{} \\
\hline & Não & 8 & 14 & 22 & & \\
\hline \multirow{2}{*}{$\begin{array}{l}\text { Conhecimento sobre a TB antes } \\
\text { do diagnóstico }\end{array}$} & Satisfatório & 14 & 16 & 30 & \multirow{2}{*}{1,08} & \multirow{2}{*}[0,67;1,73]{} \\
\hline & Precário & 29 & 38 & 67 & & \\
\hline \multirow{3}{*}{$\begin{array}{l}\text { Primeiro Serviço de Saúde } \\
\text { procurado ao perceber os } \\
\text { sintomas da TB }\end{array}$} & Atenção Básica $^{(1)}$ & 14 & 15 & 29 & \multirow{3}{*}{$\begin{array}{l}\mathrm{RP}_{1 / 2}-1,01 \\
\mathrm{RP}_{1 / 3}-1,61\end{array}$} & \multirow{3}{*}{$\begin{array}{l}{[0,62 ; 1,63} \\
{[0,75 ; 3,47}\end{array}$} \\
\hline & Pronto Atendimento ${ }^{(2)}$ & 23 & 25 & 48 & & \\
\hline & Serviços Especializados ${ }^{(3)}$ & 6 & 14 & 20 & & \\
\hline
\end{tabular}

${ }^{*} \mathrm{RP} 1 / \mathrm{n}$ equivale a razão de prevalência entre a categoria 1 em relação a categoria $\mathrm{n}$, sendo $\mathrm{n}$ variando de 2 a 5 .

\section{DISCUSSÃO}

O predomínio de TB em pessoas do sexo masculino, neste estudo, aponta a maior suscetibilidade masculina à doença e reflete os hábitos de vida e a possibilidade da mulher ser mais cuidadosa com a saúde do que o homem. Nesse sentido, verifica-se que a presença dos homens nos serviços de saúde está predominantemente ligada a motivos de doença( ${ }^{(9)}$, o que revela os papéis assumidos socialmente por homens e mulheres e as diferenças nas formas de expressar e perceber a necessidade de saúde e procurar por atendimento. Embora o sexo masculino assuma maior peso nos perfis de morbidade e mortalidade por TB, os homens atrasaram mais que as mulheres para buscar por atendimento nos serviços de saúde. Sobre esse aspecto, existem divergências na literatura mostrando que a expressão do gênero é diversificada no contexto de cada país: na Índia obteve-se esse mesmo resultado(5); na
Colômbia, não houve divergências no tempo de busca por assistência entre homens e mulheres ${ }^{(10)}$, porém na Tanzânia( ${ }^{(4)}$, por razões relacionadas à aspectos culturais que incorrem na falta de autonomia e de acesso à recursos financeiros, as mulheres demoraram mais para buscar o diagnóstico da TB.

Outro aspecto a ser considerado refere-se ao predomínio de atraso entre os doentes das faixas etárias de 18 a 29 anos, o que revela não só a tendência desses indivíduos em apoiar economicamente a família como também a influência de aspectos relacionados à acessibilidade de horários aos serviços de saúde no padrão de comportamento de busca por atendimento ${ }^{(7,11)}$. Ademais, dados da literatura têm reafirmado a maior probabilidade de indivíduos com idade avançada procurar mais por consultas 
médicas em função do acometimento por moléstias crônicas e degenerativas que necessitam de maior acompanhamento ${ }^{(12)}$. No entanto, para a faixa etária dos doentes de 50 a 59 anos, que nesse estudo demorou mais tempo para buscar por atendimento, embora sintomas clássicos da TB como tosse crônica, febre e perda de peso possam estar presentes, a coexistência de outras doenças podem ter mascarado o quadro de adoecimento tornando a percepção dos sintomas da doença mais complexa, sendo muitas vezes atribuídas a outras doenças vinculadas ao aparelho respiratório, contribuindo ao agravamento do quadro clínico pela demora do diagnóstico ${ }^{(7,12-13)}$.

Neste estudo, a demora na busca por atendimento pode ter sido influenciada pela predominância de doentes com baixa escolaridade, o que vai ao encontro de outros achados que relacionam níveis escolares à falta de conhecimento e autopercepção sobre o estado de saúde, sendo a busca por atendimento atrelada à piora do quadro clínico $^{(4-5,14)}$. Destaca-se também a existência de uma possível articulação entre os níveis de educação e renda, uma vez que se observou maior busca por atendimento entre indivíduos com baixa renda e mais anos de escolaridade ${ }^{(12)}$. Dessa maneira, não se descarta a possibilidade de interação entre as características do perfil dos doentes na definição do tempo para a busca do primeiro atendimento.

No presente estudo, aventa-se a possibilidade de que os doentes com TB tenham subvalorizado os sintomas da doença, corroborando com a importância dada à percepção da gravidade dos sintomas para ocorrência da demanda aos serviços de saúde ${ }^{(13-15)}$. O fato de um dos principais sintomas da TB pulmonar ${ }^{(5)}$, a tosse, se manifestar inicialmente de maneira mais branda ${ }^{(7,16)}$; de a sintomatologia inicial ter sido relatada como fraca; e de fumantes terem maior suscetibilidade a sintomas como a tosse e dispneia ${ }^{(17)}$; pode ter afastado a suspeita de TB ou de outra doença grave entre os doentes, levando-os a não sentirem a necessidade de procurar por atendimento em um serviço de saúde. $O$ atraso na busca por atendimento entre os doentes com TB que consumiam bebidas alcoólicas vai ao encontro de outros estudos ${ }^{(5,18)}$ e pode estar relacionado à percepção precária acerca de sua saúde, bem como à falta de hábito de procurar atendimento médico.

A elevada frequência de etilismo e tabagismo entre doentes com TB e sua interferência na eclosão e agravamento do quadro clínico da doença ${ }^{(7)}$ levantam a necessidade de os profissionais de saúde questionarem os usuários acerca dessas características comportamentais e estarem sensibilizados quanto a outras características relacionadas ao atraso do doente na busca por atendimento mostradas neste estudo, favorecendo a suspeição de um sintomático respiratório e diminuindo o atraso para a obtenção do diagnóstico e início do tratamento entre os doentes.

Em São José do Rio Preto, a não realização de controle preventivo de saúde ou consultas de rotina pelos doentes com TB, bem como a proximidade dos serviços de saúde, não se mostraram determinantes na procura por atendimento em menor tempo entre os doentes com TB, como também encontrado em outro estudo ${ }^{(6)}$, revelando que, após a percepção de um problema/necessidade, a procura por atendimento ocorre principalmente pela credibilidade atribuída ao estabelecimento e ao vínculo entre os usuários e os profissionais de determinados pontos de atenção à saúde. Assim, o uso regular de um mesmo serviço pode ser interpretado como a existência de uma porta de entrada ao sistema de saúde a cada nova necessidade/ problema ${ }^{(19)}$, independente da distância geográfica existente entre a moradia do usuário até o serviço de saúde.

Da mesma forma, o medo e desvalorização de um novo diagnóstico de TB, bem como uma experiência ruim e possível insatisfação quanto ao atendimento recebido anteriormente podem ter levado os doentes que estavam em recidiva ou retratamento a adiar a busca por atendimento. Dessa maneira, a avaliação da satisfação dos doentes quanto à assistência ofertada nos serviços de saúde é de suma relevância, uma vez que revela o modo que o usuário tem recebido essa atenção. Satisfeito com o acoIhimento, o usuário retorna ao serviço por tê-lo como referência na resolução de seus problemas/necessidades, garantindo a longitudinalidade do cuidado a cada nova necessidade de saúde percebida. Destacam-se, nesse ponto, como forma de favorecer o acesso e utilização dos serviços de saúde, a implementação de ações intersetoriais que melhorem as formas de organização de trabaIho e o fluxo de informações entre os diferentes níveis de atenção, bem como estratégias de responsabilização compartilhada entre os diversos atores sociais que promovam a participação social, direcionando a oferta da atenção de acordo com as necessidades dos usuários ${ }^{(20)}$.

Entre doentes em situação de coinfecção com o HIV, provavelmente por possuírem vínculo e já realizarem controle de saúde constante em um serviço de referência aos usuários portadores de HIV/aids do município, houve maior rapidez para procurar o serviço de saúde. Além disso, esses indivíduos conhecem seu estado de imunossupressão e manifestam mais gravemente os sintomas da TB. Contrariando esse resultado, a literatura revela maior atraso na busca pelo diagnóstico entre coinfectados ${ }^{(14)}$, o que pode estar relacionado ao medo de ser diagnosticado com TB e a presença de sinais e sintomas comuns às duas doenças, como a perda de peso, que pode influenciar na percepção da alteração de sua saúde.

Doentes com TB que relataram ter conhecimento satisfatório sobre a doença tiveram maior atraso na busca por serviços de saúde. Para uma melhor análise dessa relação, seria preciso utilizar uma ferramenta mais apropriada para medir o conhecimento dos doentes com TB, que englobasse aspectos da sintomatologia, transmissibilidade e consequências do atraso ao diagnóstico e tratamento.

A respeito da maior preferência dos doentes com TB pelos serviços de Pronto Atendimento ressalta-se que, no 
Brasil, há uma política de incentivo à construção de novas unidades, o que de certa forma colabora para a persistência de busca por atendimento nesses serviços. A dificuldade de acesso, suposição da incapacidade e desconfiança na resolução da necessidade e inexistência de vagas para atendimento nos serviços de Atenção Básica são possíveis causas que podem induzir a procura direta dos usuários aos serviços de Pronto Atendimento, corroborando com o atraso encontrado entre doentes que buscaram pelos serviços primários neste estudo. Tal comportamento não favorece a organização da demanda e do atendimento das condições crônicas, como a TB, uma vez que serviços de Pronto Atendimento não possuem a função de coordenação da assistência ${ }^{(7,13,21-22)}$.

Nesse sentido, independente do tipo de serviço de saúde procurado, a iniciativa de buscar por atendimento mostra-se fortemente atrelada ao vínculo, à busca por profissionais competentes, à satisfação com o acolhimento prestado, à confiança na experiência e à capacidade dos membros da equipe de saúde ${ }^{(19-20)}$, podendo ser realizada em mais ou menos tempo por razões geográficas, políticas, econômicas e antropológicas do contexto social do indivíduo ${ }^{(7,12,15)}$.

Dentre as limitações do estudo, destaca-se o viés de memória do doente sobre o aparecimento e identificação dos sintomas da TB. Ressalta-se que a realização das en-

\section{REFERÊNCIAS}

1. World Health Organization (WHO). Global Tuberculosis Control: surveillance, planning, financing. Geneva; 2010.

2. Maciel ELN, Golub JE, Peres RL, Hadad DJ, Fávero JL, Molino LP, et al. Delay in diagnosis of pulmonary tuberculosis at a primary health clinic in Vitoria, Brazil. Int J Tuberc Lung Dis. 2010;14(11):1403-10.

3. Sreeramareddy CT, Panduru KV, Menten J, Ende JVD. Time delays in diagnosis of pulmonary tuberculosis: a systematic review of literature. BMC Infect Dis. 2009;9(1):91-101.

4. Mfinanga SG, Mutayoba BK, Kahwa A, Kimaro G, Mtandu R, Ngadaya $E$, et al. The magnitude and factors responsible for delay in tuberculosis management in Dar es salaam, Tanzania. BMC Health Serv Res. 2008;8:158.

5. Rajeswari R, Chandrasekaran V, Suhadev M, Sivasubramaniam S, Sudha G, Renu G. Factors associated with patient and health system delays in the diagnosis of tuberculosis in South India. Int J tuberc Lung Dis. 2002;6(9):789-95.

6. Monroe AA, Gonzalez RIC, Palha PF, Sassaki CM, Ruffino-Netto $A$, Vendramini SHF, et al. Involvement of health primary care teams in the control of tuberculosis. Rev Esc Enferm USP. 2008;42(2):262-67. trevistas só foi possível através da parceria com os serviços e apoio das equipes de saúde locais na localização e agendamento dos doentes.

\section{CONCLUSÃO}

O comportamento de busca tardia por atendimento pode ser considerado grave do ponto de vista epidemiológico uma vez que prevê uma maior disseminação e agravamento da doença.

Nesse sentido, o presente estudo traz como contribuição a identificação do perfil dos usuários que atrasaram para buscar pelo primeiro atendimento, subsidiando as equipes de saúde para o reconhecimento deste, bem como para a definição de estratégias que privilegiem a desmistificação da TB na comunidade, a autopercepção precoce dos sintomas da doença entre os usuários, a redução de barreiras de acesso ao atendimento e o fortalecimento do acolhimento de modo a favorecer a busca por atendimento entre os usuários em menor tempo e a realização do diagnóstico em tempo oportuno.

Cabe destacar que algumas das características do perfil dos doentes que apresentaram atraso na busca por atendimento transcendem o setor saúde, tornando-se imprescindível a atuação intersetorial para o controle da TB.

7. Job JRPP, Gozzano JOA, Bernardes Júnior OR, Garcia RH, Miralhes OJC, Miranda MAP. Informações que antecederam o diagnóstico de tuberculose pulmonar e tempo decorrido até o início do tratamento em pacientes matriculados em Centro de Saúde, São Paulo (Brasil). Rev Saúde Pública. 1986;20(1):21-5.

8. Machado ACFT, Steffen RE, Oxlade O, Menzies D, Kritski A, Trajman A. Fatores associados ao atraso no diagnóstico da tuberculose pulmonar no estado do Rio de Janeiro. J Bras Pneumol. 2011;37(4):512-20.

9. Gomes R, Nascimento EF, Araújo FC. Por que os homens buscam menos os serviços de saúde do que as mulheres? As explicações de homens com baixa escolaridade e homens com ensino superior. Cad Saúde Pública. 2007;23(3):565-74.

10. Cáceres-Manrique FM, Orozco-Vargas LC. Demora en el diagnóstico de tuberculosis pulmonar en una región de Colombia. Rev Salud Pública. 2008;10(1):94-104.

11. Eastwood SV, Hill PC. A gender focused qualitative study of barriers to accessing tuberculosis in The Gambia, West Africa. Int J Tuberc Lung Dis. 2004;8(1):70-5.

12. Mendoza-Sassi R, Béria JU, Barros AJD. Outpatient health service utilization and associated factors: a population-based study. Rev Saúde Pública. 2003;37(3):372-78.
Atraso na procura pelo primeiro atendimento para o diagnóstico da tuberculose Wysocki AD, Ponce MAZ, Scatolin BE, Andrade RLP, Vendramini SHF, Ruffino-Netto A, Villa TCS 
13. Maior ML, Guerra RL, Cailleaux-Cezar M, Conde MB. Tempo entre o início dos sintomas e o tratamento de tuberculose pulmonar em um município com elevada incidência da doença. J Bras Pneumol. 2012;38(2):202-9.

14. Mesfin MM, Newell JN, Walley JD, Gessessew A, Madeley RJ. Delayed consultation among pulmonary tuberculosis patients: a cross sectional study of 10 DOTS districts of Ethiopia. BMC Public Health. 2009;9:53.

15. Mendoza-Sassi R, Béria JU, Fiori N, Bortolotto A. Prevalência de sinais e sintomas, fatores sociodemográficos associados e atitude frente aos sintomas em um centro urbano no Sul do Brasil. Rev Panam Salud Pública. 2006;20(1):22-8.

16. Nogueira JA, Ruffino Netto A, Monroe AA, Gonzales RIC, Villa TCS. Busca ativa de sintomáticos respiratórios no controle da tuberculose na percepção do agente de saúde. Rev Eletr Enferm [Internet] 2007[ citado 2011 ago. 19];9(1):10618. Disponível em: http://www.fen.ufg.br/revista/v9/n1/ v9n1a08.htm

17. Basnet R, Hinderaker SG, Enarson D, Malla P, Morkve O. Delay in the diagnosis of tuberculosis in Nepal. BMC Public Health. 2006;9:236-45.
18. Van Der Werf MJ, Chechulin Y, Yegorova OB, Marcinuk T, Stopolyanskiy A, Voloschuk $\mathrm{V}$, et al. Health care seeking behaviour for tuberculosis symptoms in Kiev City, Ukraine. Int J Tuberc Lung Dis. 2006;10(4):390-5.

19. Ramos DD, Lima MADS. Acesso e acolhimento aos usuários em uma unidade de saúde de Porto Alegre, Rio Grande do Sul, Brasil. Cad Saúde Pública. 2003;19(1):27-34.

20. Ponce MAZ, Vendramini SHF, Santos MR, Santos MLSG, Scatena LM, Villa TCS. Vínculo profissional/doente no tratamento da tuberculose: desempenho da atenção básica em município do interior paulista. Rev Latino Am Enferm. 2011;19(5):1222-9.

21. Oliveira LH, Mattos RA, Souza AIS. Cidadãos peregrinos: os "usuários" do SUS e os significados de sua demanda a prontos-socorros e hospitais no contexto de um processo de reorientação do modelo assistencial. Ciênc Saúde Coletiva. 2009;14(5): 1929-38.

22. Marques GQ, Lima MAD. Demandas de usuários a um serviço de pronto atendimento e seu acolhimento ao sistema de saúde. Rev Latino Am Enferm. 2007;15(1):13-9.

\section{Agradecimentos}

Aline Ale Beraldo, Profa Dra aline Aparecida Monroe, Gabriela Tavares Magnabosco, Profạ Drª Lucia Marina Scatena, Profa Dra Maria de Lourdes Sperli Geraldes Santos, Maria Eugênia Firmino Brune, Reinaldo Antônio da Silva Sobrinho, Dra Maria Rita de Cássia Oliveira Cury, Tiemi Arakawa. 